\title{
Broadband Cherenkov Radiation by Using Group-velocity-matching in Index-guiding Photonic Crystal Fiber
}

\author{
Zeng, Xianglong; Guo, Hairun; Wang, Shaofei; Bache, Morten
}

Published in:

CLEO/Europe-IQEC 2013 - European Conference on Lasers and Electro-Optics \& the International Quantum Electronics Conference

Publication date:

2013

Link back to DTU Orbit

Citation (APA):

Zeng, X., Guo, H., Wang, S., \& Bache, M. (2013). Broadband Cherenkov Radiation by Using Group-velocitymatching in Index-guiding Photonic Crystal Fiber. In CLEO/Europe-IQEC 2013 - European Conference on Lasers and Electro-Optics \& the International Quantum Electronics Conference IEEE.

\section{General rights}

Copyright and moral rights for the publications made accessible in the public portal are retained by the authors and/or other copyright owners and it is a condition of accessing publications that users recognise and abide by the legal requirements associated with these rights.

- Users may download and print one copy of any publication from the public portal for the purpose of private study or research.

- You may not further distribute the material or use it for any profit-making activity or commercial gain

- You may freely distribute the URL identifying the publication in the public portal 


\section{Broadband Cherenkov Radiation by Using Group-velocity-matching in Index-guiding Photonic Crystal Fiber}

Xianglong Zeng, ${ }^{1,2}$ Shaofei Wang, ${ }^{2}$ Hairun Guo, ${ }^{1}$ Morten Bache ${ }^{1}$

1. Ultrafast Nonlinear Optics group, DTU Fotonik, Technical University of Denmark, DK-2800 Kgs. Lyngby, Denmark

2. Key Laboratory of Special Fiber Optics and Optical Access Networks, Shanghai University, Shanghai 200072, China

Dispersive waves (DW) are radiated when temporal solitons are perturbed by higher-order dispersion (HOD). This is also called optical Cherenkov radiation (OCR). Currently among much efforts of generating optical octavespanning supercontinuum (SCG), OCR has become an efficient nonlinear frequency conversion in the blue edge of SCG and blue-shifting the edge with the soliton self-frequency shift(SSFS) by the Raman effect [1]. Moreover, a sharp switching of SSFS can occur across a normal group velocity dispersion (GVD) region sandwiched in the anomalous GVD regions, known as soliton spectral tunneling (SST) effect [2]. Its mechanism was attributed to the phase-matching (PM) between the soliton and linear DW in the anomalous GVD. Here we argue first that groupvelocity (GV) matching is another necessary condition for such SST effect and present a detailed analysis on the broadband OCR by employing GV-matching in the index-guiding photonic crystal fibers (PCFs). This approach is expected to efficiently generate ultrashort pulses in the near- and mid-IR.
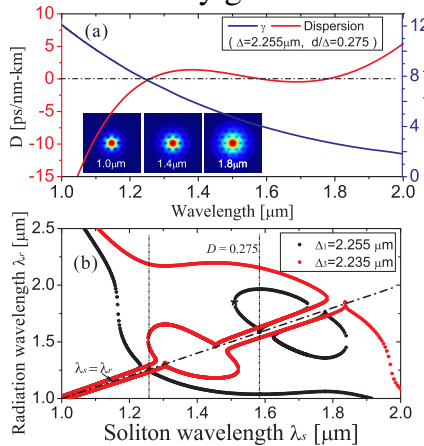

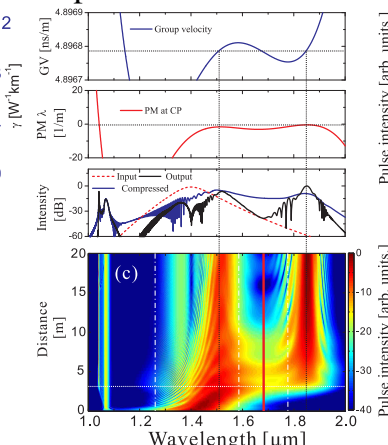

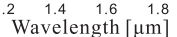

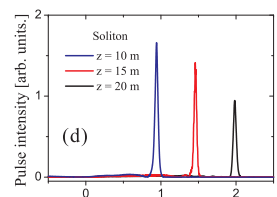

Time delay $[\mathrm{ps}]$

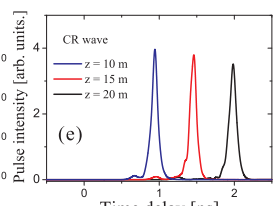

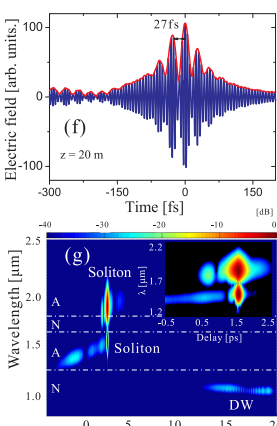

Delay $[\mathrm{ps}]$

Fig. 1 (a) Chromatic dispersion and effective nonlinear coefficient $\gamma$ versus wavelength (insets are mode field distributions). (b) Phase matching curves between soliton and its radiation wavelengths, $\star$ indicate the critical point (CP). (c) Spectra evolution of 25 fs (FWHM) input soliton $(\mathrm{N}=1)$ at the pump wavelength of $1.40 \mu \mathrm{m}$ under the dispersion profile $\left(\Delta_{1}\right)$. (d) and (e) are soliton and OCR pulses by using a lowpass filter cut at $1.68 \mu \mathrm{m}$ (red line in (c)). (f) The electric field of output pulse by solving the NWEF. (g) Spectrogram of the output pulse.

The PM condition $\Delta \beta \equiv \beta_{s}\left(\omega_{s}\right)-\beta\left(\omega_{r}\right)=0$ determines the radiation frequencies, where $\beta_{s}\left(\omega_{j}\right)=\beta\left(\omega_{j}\right)+$ $\left(\omega-\omega_{j}\right) / v_{g}\left(\omega_{j}\right)+q_{j}$ reflects the nondispersive nature of the soliton at the center frequency $\omega_{j}, \beta(\omega)$ is the propagating constant and $v_{g}\left(\omega_{j}\right)=\frac{\partial \beta}{\partial \omega}||_{\omega=\omega_{j}}^{-1}$ is the group velocity, $j=r, s$ represents the radiation or soliton wave. Since the OCR in SST process tends to be a soliton state in the anomalous GVD regime, the PM condition instead becomes $\beta_{s}\left(\omega_{s}\right)=\beta_{s}\left(\omega_{r}\right)$ for any frequencies, i.e., additionally requiring $v_{g}\left(\omega_{s}\right)=v_{g}\left(\omega_{r}\right)$. Fig. 1(a) shows the dispersion profile with controlled three zero-dispersion wavelengths $\left(\lambda_{1}<\lambda_{2}<\lambda_{3}\right)$ in the small-core PCFs, in which it is possible to observe SST effect by launching a soliton in the first anomalous GVD regime $\left(\lambda_{1}<\lambda_{s}<\lambda_{2}\right)$. Fig. 1(b) shows two PM conditions for different dispersion profiles. Between $\lambda_{1}$ and $\lambda_{2}$ (vertical black lines), the one with black dots has a so-called critical point (CP) of the soliton wavelength, where two radiation bands are degenerate, indicating that $\beta_{s}(\omega)$ is the tangent line of $\beta(\omega)$ at both $\omega_{s}$ and $\omega_{r}$, i.e., satisfying $v_{g}\left(\omega_{s}\right)=v_{g}\left(\omega_{r}\right)$.

We model the nonlinear pulse propagation by using a nonlinear wave equation in the frequency domain (NWEF) [3], which directly describes the electric field dynamics. Fig. 1(c) shows a simulation when the input soliton pulse is red-shifted through Raman effects to the CP wavelength $\left(\lambda_{p}=1.51 \mu \mathrm{m}\right)$, a strong and broadband radiation is emitted at $1.85 \mu \mathrm{m}$, where the radiation is GV matched to the soliton pulse. Besides, two narrow and weak radiations at $1.07 \mu \mathrm{m}$ and $1.04 \mu \mathrm{m}$ are emitted within a short interaction length with a large GV mismatch.

Moreover, by expanding the PM condition at the radiation wavelength: $\frac{\partial(\Delta \beta)}{\partial \lambda_{r}}=\frac{4 \pi c}{\lambda^{2}}\left[1 / v_{g}\left(\omega_{s}\right)-1 / v_{g}\left(\omega_{r}\right)\right]$, we can see that GV matching enables larger bandwidths. This is easily understood by recalling that for a $\chi^{(2)}$ nonlinear process, GV matching between the interaction pulses means larger bandwidths for conversion process and longer interaction lengths. Fig. 1(d) and (e) show the individual soliton and OCR pulses by filtering the spectrum cut at $1.68 \mu \mathrm{m}$. They follow the soliton state after the tunneling process. The electric field of the output pulse is calculated by using the NWEF as shown in Fig. 1(f). GV matching between the radiation and the soliton leads to the beating modulation in the electric field and the beating period is $27 \mathrm{fs}$, which corresponds to their frequency difference of temporally overlapped pulses. The spectrogram of the output pulse is shown in Fig. 1(g): a stable soliton state keeps with the same GV as the red-shifted soliton.

\section{References}

[1] J. M. Dudley and J. R. Taylor, Supercontinuum Generation in Optical Fibers (Cambridge University Press, 2010).

[2] B. Kibler, P.-A. Lacourt, F. Courvoisier and J.M. Dudley, "Soliton spectral tunnelling in photonic crystal fibre with sub-wavelength core defect," Electron. Lett. 43, 967-968 (2007).

[3] H. Guo, X. Zeng, B. B. Zhou, and M. Bache, "Electric-field modeling and self-steepening counterbalance of cascading nonlinear soliton pulse compression,” arXiv:1210.5903 (2012). 